\title{
A New Step in the Solution of the Szökefalvi-Nagy Problem
}

\author{
Vladimir G. Boltyanski* \\ Department of Mathematics, University of Washington, \\ Seattle, WA 98195, USA \\ Communicated by Victor Klee
}

\begin{abstract}
A description of vector systems $H \subset R^{d}$ that satisfy the condition md $H=2$ is given. With the help of this description the main result is obtained. It consists in a listing of all compact, 2-Helly-dimensional, convex bodies $M \subset R^{d}$. The listing is made in terms of polar bodies $M^{*}$.
\end{abstract}

\section{Introduction}

Let $M \subset R^{d}$ be a compact, convex body, and let $T(M)$ be the family of all its translates. By him $T(M)$ we denote the Helly dimension of the family, that is, the least of integers $m \geq 0$ that have the following property: For every collection of sets $M_{1}, \ldots, M_{s} \in T(M), s>m+1$, if each $m+1$ of them has a nonempty intersection, then the intersection $M_{1} \cap \cdots \cap M_{s}$ of all sets taken is also nonempty.

It is easily shown that if $M \subset R^{d}$ is a $d$-dimensional parallelotope, then $\operatorname{him} T(M)=1$. This means that if $M_{1}, \ldots, M_{s}, s>2$, are translates of a parallelotope, such that every two of them have a nonempty intersection, then $M_{1} \cap \cdots \cap M_{s} \neq \varnothing$. The famous Hungarian mathematician Szökefalvi-Nagy has proved [1] that the converse assertion is also true:

Theorem 1 (Szökefalvi-Nagy). If $M \subset R^{d}$ is a compact convex body and $\operatorname{him} T(M)=1$, then $M$ is a parallelotope. Russia.

* Current address: Institute of Systems Research, Prosp. 60-years of October 9, Moscow 117312, 
This interesting result leads us to the following problem of combinatorial geometry:

The Szökefalvi-Nagy Problem. To find all compact, convex bodies $M \subset R^{d}$ for which him $T(M)=s$ where $s$ is a given integer $(2 \leq s \leq d)$.

We indicate partial results that were obtained in this direction. In order to state these results we recall some notions.

Definition 1. Let $M_{1}, \ldots, M_{k}$ be convex sets in $R^{d}$. The set of all points $x_{1}+\cdots+x_{k}$, where $x_{1} \in M_{1}, \ldots, x_{k} \in M_{k}$, is said to be the vector sum (or Minkowski's sum) of the convex sets $M_{1}, \ldots, M_{k}$. If there exist subspaces $L_{1}, \ldots, L_{k}$ of $R^{d}$, such that $R^{d}=L_{1} \oplus \cdots \oplus L_{k}$ and, for every $i=1, \ldots, k$, a translate of $M_{i}$ is contained in $L_{i}$, then the vector sum $M_{1}+\cdots+M_{k}$ is said to be the direct vector sum and is denoted by $M_{1} \oplus \cdots \oplus M_{k}$.

The following theorem was proved in [2].

Theorem 2 (Boltyanski). Let $M \subset R^{d}$ be a compact, centrally symmetric, convex body. If him $T(M)=2$, then $M$ is a direct vector sum of convex sets each of which has dimension $\leq 2$.

We are now going to formulate the results that were established in [3] and [4], but first we describe two classes of the convex bodies (see [5]-[7]).

Definition 2. A convex polytope $M \subset R^{d}$ is said to be a zontope if it may be represented as a vector sum (in the general case, nondirect) of a finite number of closed segments. A compact, convex body $M \subset R^{d}$ is said to be a zonoid if it is a limit (in the Hausdorff metric) of a convergent sequence of zontopes,

Theorem 3 (Baladze). Let $M \subset R^{d}$ be a zonoid (in particular, a zonotope). The inequality him $T(M) \leq s$ is true if and only if $M$ is a direct vector sum of convex sets, each of which has dimension $\leq s$.

The aforesaid theorems are in accordance with the following:

Direct Sum Conjecture. Let $M \subset R^{d}$ be a compact, convex body. If him $T(M) \leq s$, then $M$ is a direct vector sum of convex sets, each of which has dimension $\leq s$.

More exactly, in Theorems 1-3 a class $K$ of convex bodies in $R^{d}$ and a subset $S=\{1,2, \ldots, d\}$ are indicated, such that for every convex body $M \subset K$ and every $s \in S$ the direct sum conjecture holds. Indeed, in Theorem $1 K$ is the class of all convex bodies and $S=\{1\}$. Furthermore, in Theorem $2 \mathrm{~K}$ is the class of all centrally symmetric convex bodies and $S=\{1,2\}$. Finally, in Theorem $3 K$ is the class of all zonoids in $R^{d}$ (in particular, zonotopes) and $S=\{1,2, \ldots, d\}$. In each of these cases the direct sum conjecture is confirmed. 
Besides that, there are some results on the Szökefalvi-Nagy problem which fall outside the framework of the direct sum conjecture. In [8] Boltyanski and Chabukiani have listed all convex bodies in $R^{3}$ that satisfy the condition $\operatorname{him} T(M)=2$ (without the assumption of central symmetry). In [9] Kincses has listed all centrally symmetric convex bodies in $R^{d}$ that satisfy the conditions him $T(M)=3$ or him $T(M)=4$. Furthermore, we mention one more result [2], [10] that gives a formal algebraic solution of the Szökefavi-Nagy problem in the general case. For this purpose we need several definitions.

Definition 3. Vectors $a_{0}, \ldots, a_{m} \in R^{d}$ are said to be minimally dependent if they are the vertices of an $m$-dimensional simplex $T$, such that the origin 0 belongs to the relative interior relint $T$ of $T$. Equivalently, the vectors $a_{0}, \ldots, a_{m}$ are minimally dependent if and only if (i) they are positively dependent:

$$
\lambda_{0} a_{0}+\cdots+\lambda_{m} a_{m}=0, \quad \lambda_{0}>0, \ldots, \lambda_{m}>0
$$

and (ii) any $m$ of them (consequently, each $m$ of them) are linearly independent.

Definition 4. Let $H$ be a nonempty subset of the unit sphere $S^{d-1} \subset R^{d}$. By md $H$ we denote the largest of integers $m$, such that there is in $H$ a system of $m+1$ minimally dependent vectors. (If there is no system of minimally dependent vectors in $H$, then md $H=0$.) Furthermore, let $M \subset R^{d}$ be a convex body that does not coincide with $R^{d}$. The set of all unit vectors $x \in S^{d-1}$ that are outward normals of the body $M$ at its regular boundary points will be denoted by $H(M)$, and the number md $H(M)$ will be denoted by $\operatorname{md}$. We recall that a boundary point $a$ of a body $M$ is said to be regular if there exists only one hyperplane $L$ through $a$ that supports the body $M$.

Let us note that if a convex body $M \subset R^{d}$ is compact, then the set $H(M)$ is not one-sided, that is, there is no closed hemisphere $P \subset S^{d-1}$ that contains $H(M)$ (or, what is the same, there exists no vector $p \in S^{d-1}$, such that $\langle p, x\rangle \leq 0$ for every $x \in H(M)$ ).

Theorem 4 (Boltyanski). Let $M \subset R^{d}$ be a compact, convex body. The Helly dimension of the family $T(M)$ is equal to $\operatorname{md} M$, that is, $\operatorname{him} T(M)=\operatorname{md} M$.

This theorem is an essential step for a complete geometrical solution of the Szökefalvi-Nagy problem, that is, for a description of all compact, convex bodies $M \subset R^{d}$ with him $T(M)=r$ in geometrical terms (for example, to be a direct vector sum, to be a parallelotope, to have dimension $\leq r$, and so on).

Besides that, we indicate two theorems (that are, at least for us, auxiliary ones). The first of them was established by Givalevich [11], the second is a trivial consequence of a classical theorem of the theory of convex sets.

Theorem 5 (Givalevich). If a vector system $H \subset R^{d}$ is not one-sided, then md $H=\operatorname{md~} \mathrm{cl} H$. 
Definition 5. Let $M \subset R^{d}$ be a compact, convex body. A boundary point $x \in$ bd $M$ is said to be an extremal point of $M$ if there exists no segment $I \subset M$, such that $x \in$ relint $I$. The set of all extremal points of the convex body $M$ is denoted by ext $M$.

Definition 6. Let $M \subset R^{d}$ be a set of vectors. By norm $E$ we denote the set of all normed nonzero vectors from $E$, that is, $y \in$ norm $E$, if and only if there exists a vector $x \in E$, such that $x \neq 0$ and $y=x /|x|$.

Definition 7. Let $X \subset R^{d}$ be a convex set and let $a \in R^{d}$. The polar set $X_{a}^{*}$ of the set $X$ with respect to the point $a$ is defined by

$$
X_{a}^{*}=\left\{y \in R^{d}:\langle x-a, y\rangle \leq 1 \text { for all } x \in X\right\} \text {. }
$$

Theorem 6. Let $M \subset R^{d}$ be a compact, convex body and let $a \in$ int $M$. Then the inclusions $H(M) \subset$ norm ext $M_{a}^{*} \subset \mathrm{cl} H(M)$ are true.

The main purpose of the paper is to state and to prove a theorem that contains a listing of all compact, convex bodies $M \subset R^{d}$ with $\operatorname{him} T(M)=2$ (or, what is the same, md $M=2$ ). This result will be obtained in the general case, that is, without the assumption of central symmetry. However, we use a new approach to a solution of the Szökefalvi-Nagy problem. This approach (applied in [3], [4], [8], and, in particular, [9]) involves describing not the bodies $M$ with md $M=r$, but their polar bodies $M^{*}$ instead.

\section{Statements of the Results}

First we give a description of non-one-sided vector systems $H \subset R^{d}$ that satisfy the condition md $H=2$. With the help of this description we obtain a listing of compact, convex bodies in $R^{d}$ with md $M=2$ (in terms of their polars).

Definition 8. A vector system $H \subset S^{d-1}$ is said to be decomposed if there exists a direct decomposition $R^{d}=L_{1} \oplus \cdots \oplus L_{s}$ (where $\operatorname{dim} L_{i}<d$ for all $i=1, \ldots, s$ ), such that $H \subset L_{1} \cup \cdots \cup L_{s}$.

Definition 9. A vector system $H \subset R^{d}$ is said to be 2-system if it is non-one-sided and satisfies the condition md $H=2$.

By virtue of this definition, for every 2-system $H \subset S^{d-1}$ there exists a direct decomposition $R^{d}=L_{1} \oplus \cdots \oplus L_{s}(s \geq 1)$, such that $H \subset L_{1} \cup \cdots \cup L_{s}$ and each vector system $H \cap L_{i}$ is an indecomposable 2-system. So it is sufficient to describe all indecomposable 2-systems.

Furthermore, according to the following theorem, it is sufficient to find all maximal 2-systems (since if a 2-system $H \subset L_{i}$ is indecomposable, then a maximal 2-system in $L_{i}$ that contains $H$ is indecomposable, too.) 
Theorem 7. Each indecomposable 2-system $H \subset R^{d}$ is contained in a maximal indecomposable 2-system.

Proof. By virtue of Theorem 5, each 2-system is contained in a closed 2-system (because each 2-system $H \subset S^{d-1}$ is not one-sided). So it is sufficient to consider only closed 2-systems. Furthermore, for every 2-system $H \subset S^{d-1}$ its convex hull conv $H$ is a convex body in $R^{d}$ that is uniquely defined by $H$. This means that we must find a maximal element in the family (ordered by inclusion) consisting of all convex bodies conv $H^{\prime}$ for closed 2-systems $H^{\prime}$, each of which contains the given 2-system $H$. For closed, convex bodies in $R^{d}$ an application of the Zorn lemma does not require use of the Zermelo axiom. So it is sufficient to prove that each chain $F^{\prime} \subset F$ is bounded. Indeed, the set

$$
H_{1}=\mathrm{cl} \bigcup_{H^{\prime} \in F^{*}} H^{\prime}
$$

is, obviously, a closed 2-system that satisfies the inequality $H^{\prime} \leq H_{1}$ for all $H^{\prime} \in F^{\prime}$. This completes the proof.

Now we introduce notions that help us describe all maximal 2-systems.

Definition 10. Let $H \subset S^{d-1}$ be a non-one-sided vector system. A vector $e \in R^{d}$ is said to be particular with respect to $H$, if it cannot be represented as a positive linear combination of vectors from $H \sim\{e\}$.

It is evident that if $e \in R^{d}$ is a particular vector with respect to a 2-system $H$, then $e \in H$ and $H \sim\{e\} \subset P$, where $P$ is a closed half-space with boundary hyperplane through the origin.

In the remaining part of this section theorems will be only stated. The proofs are contained in the following sections.

Theorem 8. Let $H \subset S^{d-1}$ be an indecomposable 2-system, $d \geq 3$. If $H$ contains no particular vector, then $d=4$ and the system has (in a basis $e_{1}, e_{2}, e_{3}, e_{4}$ of the space $R^{4}$ ) the form $H=H^{(0)}$, where

$$
\begin{aligned}
& H^{(0)}=\operatorname{norm}\left\{e_{1}, e_{2}, e_{3}, e_{4},-e_{1}-e_{2},-e_{1}-e_{3},\right. \\
&\left.-e_{2}-e_{4},-e_{3}-e_{4}, e_{1}+e_{2}+e_{3}+e_{4}\right\} .
\end{aligned}
$$

Theorem 9. Let $H \subset S^{d-1}$ be a maximal, indecomposable 2-system that contains, at least, one particular vector, $d \geq 3$. We denote by $H^{\Lambda}$ the set of all particular vectors of the system $H$. Then $H^{\Delta}=\left\{e_{1}, \ldots, e_{k}\right\}$, where $e_{1}, \ldots, e_{k}$ are linearly independent. If $k>1$ we denote by $F_{i j}$ the angle $F_{i j}=\left\{v: v=x_{i} e_{i}+x_{j} e_{j} ; x_{i} \leq 0, x_{j} \leq 0\right\}$. Then

$$
H=\operatorname{norm}\left(\left\{e_{1}, \ldots, e_{d}\right\} \cup\left(\bigcup_{i<j \leq d} F_{i j}\right)\right) \quad \text { for } k=d
$$


Further, let $k<d$. Then there exist vectors $e_{k+1}, \ldots, e_{d}$ and a map

$$
\varphi:\{k+1, \ldots, d\} \rightarrow\{1, \ldots, k\}
$$

such that $e_{1}, \ldots, e_{d}$ is a basis in $R^{d}$ (not necessarily orthonormal) and, denoting by $\Pi_{l, i}$ the closed half-plane $\left\{v: v=x_{l} e_{l}+x_{i} e_{i} ; x_{i} \leq 0\right\}$, the following relations are true:

$$
\begin{aligned}
& H=\operatorname{norm}\left(\left\{e_{1}\right\} \cup\left(\bigcup_{l>1} \Pi_{i, 1}\right)\right) \quad \text { for } k=1, \\
& H=\operatorname{norm}\left(\left\{e_{1}, e_{2}\right\} \cup F_{12} \cup\left(\bigcup \prod_{l, \varphi(l)}\right)\right) \text { and } \varphi \text { is onto } \quad \text { for } k=2, \\
& H=\operatorname{norm}\left(\left\{e_{1}, \ldots, e_{k}\right\} \cup\left(\bigcup_{i<j \leq k} F_{i j}\right) \cup\left(\bigcup_{i>k} \Pi_{l, \varphi(l)}\right)\right) \quad \text { for } 2<k<d
\end{aligned}
$$

Remark. We may suppose (using a suitable permutation of the indices $1, \ldots, d$ ) that the function $\varphi$ is nonstrictly increasing and the inverse images $\varphi^{-1}(l)$ satisfy the condition $\left|\varphi^{-1}(1)\right| \geq \cdots \geq\left|\varphi^{-1}(k)\right|$. Under these conditions the function $\varphi$ is defined uniquely by the maximal indecomposable 2 -system $H$.

Theorems 8 and 9 give a complete listing of all maximal indecomposable 2 -systems. So, in $R^{3}$ we have only two types of maximal indecomposable 2-systems:

$$
\begin{aligned}
& H=\operatorname{norm}\left(\left\{e_{1}\right\} \cup \prod_{21} \cup \prod_{31}\right) \quad(k=1), \\
& H=\operatorname{norm}\left(\left\{e_{1}, e_{2}, e_{3}\right\} \cup F_{12} \cup F_{13} \cup D_{23}\right) \quad(k=3)
\end{aligned}
$$

(we note that the case $k=2$ is nonrealizable because there is no mapping of the set $\{3\}$ onto $\{1,2\}$ ). This result was announced in [8].

Further, in $R^{4}$ we have five types of maximal indecomposable 2-systems:
(A) $\quad H=\operatorname{norm}\left(\left\{e_{1}\right\} \cup \Pi_{21} \cup \Pi_{31} \cup \Pi_{41}\right) \quad(k=1)$,
(B) $\quad H=\operatorname{norm}\left(\left\{e_{1}, e_{2}\right\} \cup F_{12} \cup \Pi_{31} \cup \Pi_{42}\right) \quad(k=2)$,
(C) $H=\operatorname{norm}\left(\left\{e_{1}, e_{2}, e_{3}\right\} \cup F_{12} \cup F_{13} \cup F_{23} \cup \Pi_{41}\right) \quad(k=3)$,
(D) $H=\operatorname{norm}\left(\left\{e_{1}, e_{2}, e_{3}, e_{4}\right\} \cup\left(\bigcup_{i<j \leq 4} F_{i j}\right)\right) \quad(k=4)$,
(E) $H=H^{(0)} \quad$ (see Theorem 8).

This result is announced in a new paper by Boltyanski and Chabukiani (to appear in Soobtsheniya AN GSSR, Tbilisi, 1992). 
Finally, we formulate the following theorem that contains a listing of all convex, compact, indecomposable bodies $M \subset R^{d}$ with md $M=2$. In the statement of the theorem we preserve the notations of Theorem 9 (see also the Remark after Theorem 9).

Theorem 10. Let $M \subset R^{d}$ be a compact, convex body; $d \geq 3$. Let us suppose that $0 \in$ int $M$ and $M$ is not decomposable into a direct vector sum of convex sets, each of which has dimension $<d$. The inequality md $M \leq 2$ holds in and only in the following cases:

(A) There exist a basis $e_{1}, \ldots, e_{d}$ in $R^{d}$ and closed, two-dimensional convex sets $B_{i j}(i<j \leq d)$, such that

(i) $B_{i j} \subset \operatorname{lin}\left(e_{i}, e_{j}\right)$ and ext $B_{i j} \subset\left\{e_{i}, e_{j}\right\} \cup F_{i j}$,

(ii) $M^{*}=\operatorname{conv}\left(\bigcup_{i<j \leq d} B_{i j}\right)$.

(B) There exist a basis $e_{1}, \ldots, e_{d}$ in $R^{d}$ and closed, two-dimensional convex sets $C_{2}, \ldots, C_{d}$, such that

(i) $C_{l} \subset \operatorname{lin}\left(e_{1}, e_{l}\right)$ and ext $C_{l} \subset\left\{e_{1}\right\} \cup \Pi_{l, 1}$ for $l=2, \ldots, d$,

(ii) $M^{*}=\operatorname{conv}\left(C_{2} \cup \cdots \cup C_{l}\right)$.

(C) There exist a basis $e_{1}, \ldots, e_{d}$ in $R^{d}$, an integer $k(2 \leq k<d)$, a map $\varphi:\{k+1, \ldots, d\} \rightarrow\{1, \ldots, k\}$ (that is onto in the case $k=2)$, and closed, two-dimensional convex sets $B_{i j}, C_{l}(i<j \leq k ; l>k)$, such that

(i) $B_{i j} \subset \operatorname{lin}\left(e_{i}, e_{j}\right)$ and ext $B_{i j} \subset\left\{e_{i}, e_{j}\right\} \cup F_{i j}$,

(ii) $C_{l} \subset \operatorname{lin}\left(e_{l}, e_{\varphi(l)}\right)$ and ext $C_{l} \subset\left\{e_{\varphi(l)}\right\} \cup \Pi_{l, \varphi(l)}$.

(D) $d=4$ and there exists a basis $e_{1}, e_{2}, e_{3}, e_{4}$ in $R^{4}$, such that $H(M)=H^{(0)}$ (see Theorem 8).

\section{Auxiliary Propositions}

In this section we prove lemmas that are necessary for the proofs of Theorems $8-10$.

Lemma 1. Let $a_{0}, a_{1}, \ldots, a_{m}$ be positively dependent vectors. If they are not minimally dependent, then it is possible to choose among $a_{1}, \ldots, a_{m}$ fewer than $m$ vectors, such that $a_{0}$ and the chosen vectors are minimally dependent.

Proof. Let $\alpha_{0} a_{0}+\cdots+\alpha_{m} a_{m}=0$ be a positive dependence. Since $a_{0}, \ldots, a_{m}$ are not minimally dependent, then the vectors $a_{1}, \ldots, a_{m}$ are linearly dependent. Let

$$
\lambda_{1} a_{1}+\cdots+\lambda_{m} a_{m}=0
$$

be a nontrivial dependence (among the coefficients there may be positives, as well as negatives or zeros). Without loss of generality we may suppose that $\lambda_{1}>0$. If $\varepsilon>0$ is small enough, then the dependence

$$
\alpha_{0} a_{0}+\alpha_{1} a_{1}+\cdots+\alpha_{m} a_{m}-\varepsilon\left(\lambda_{1} a_{1}+\cdots+\lambda_{m} a_{m}\right)=0
$$


is positive, that is, $\alpha_{1}-\varepsilon \lambda_{1}>0, \ldots, \alpha_{m}-\varepsilon \lambda_{m}>0$. Let $\varepsilon_{0}$ be the exact upper bound of the numbers $\varepsilon>0$ for which the dependence (5) is positive (the number $\varepsilon_{0}$ exists, since, for $\varepsilon>\alpha_{1} / \lambda_{1}$, the dependence (5) is not positive). Thus for $\varepsilon=\varepsilon_{0}$ at least one of the coefficients in (5) turns to zero. So, substituting $\varepsilon=\varepsilon_{0}$ we obtain from (5) a positive dependence between $a_{0}$ and some of the vectors $a_{1}, \ldots, a_{m}$, and moreover the number of summands in the dependence obtained is less than earlier. Continuing this process, we obtain, finally, a minimal dependence between $a_{0}$ and some of the vectors $a_{1}, \ldots, a_{m}$. This completes the proof.

Lemma 2. Let $M \subset S^{d-1}$ be a 2-system. Let, furthermore, $L$ be a subspace, such that $0<\operatorname{dim} L<d$ and each vector $v \in L$ is nonparticular with respect to the vector system $H \cap L$. Then the system $H$ is decomposable.

Proof. Let $K$ be the set of all nonnegative linear combinations of the vectors from $H \sim L$. Then $K$ is a convex cone with apex 0 . First we prove that $L \cap K=\{0\}$. Let us suppose, counterwise, that there exists a nonzero vector $v_{0} \in L \cap K$. Each nonzero vector $v \in L \cap K$ is representable as a positive linear combination of several vectors from $H \sim L$. Let $m$ be the least of the integers, such that a nonzero vector $v_{0} \in L \cap K$ is positively representable through $m$ vectors from $H \sim L$, and $v_{0}=\lambda_{1} a_{1}+\cdots+\lambda_{m} a_{m}$ be the positive dependence. Then $m \geq 2$ and no nontrivial positive linear combination of fewer than $m$ of the vectors $a_{1}, \ldots, a_{m}$ belongs to $L$. Besides that, the vectors $-v_{0}, a_{1}, \ldots, a_{m}$ are minimally dependent (by virtue of Lemma 1). Since, 'urthermore, $-v_{0} \in L$ is a nonparticular vector with respect to the vector system $H \cap L$, we have $-v_{0}=\mu_{1} b_{1}+\cdots+\mu_{k} b_{k}$, where $b_{1}, \ldots, b_{k} \in(H \cap L) \sim\left\{-v_{0}\right\}$ and all the coefficients are positive $(k \geq 2)$. We may suppose that the vectors $b_{1}, \ldots, b_{k}$ are linearly independent (see Lemma 1). So we obtain the positive dependence

$$
\lambda_{1} a_{1}+\cdots+\lambda_{m} a_{m}+\mu_{1} b_{1}+\cdots+\mu_{k} b_{k}=0
$$

with not less than four summands. Moreover, the vectors $a_{1}, \ldots, a_{m-1}, b_{1}, \ldots, b_{k}$ are linearly independent (indeed, if $\gamma_{1} a_{1}+\cdots+\gamma_{m-1} a_{m-1}+v_{1} b_{1}+\cdots+v_{k} b_{k}=0$, then $\gamma_{1} a_{1}+\cdots+\gamma_{m-1} a_{m-1} \in L$; consequently, $\gamma_{1}=\cdots=\gamma_{m-1}=0$, and then $v_{1}=\cdots=v_{k}=0$ ). This means that the vectors $a_{1}, \ldots, a_{m}, b_{1}, \ldots, b_{k}$ are minimally dependent, and, hence, md $H \geq 3$, contradicting the condition of the lemma.

The contradiction obtained shows that $L \cap K=\{0\}$. We now prove that $K=$ aff $K$, that is, $K$ is a subspace. Let us suppose that $K \neq$ aff $K$. Then $0 \notin$ relint $K$ and, consequently, the convex cones $L, K$ with common apex 0 are separated. This means that there exists a hyperplane $\Gamma$ through the origin, such that $L \subset \Gamma$ and $K \subset P$ where $P$ is a closed half-space with boundary $\Gamma$. Consequently, $H=(H \cap L) \cup(H \sim L) \subset L \cup K \subset P$, contradicting the assumption that $H$ is a 2 -system (and, hence, $H$ is non-one-sided).

So, $K=$ aff $K$, that is, $K$ is a subspace of $R^{d}$. By virtue of the relations $L \cap K=\{0\}, H \subset L \cup K$, we conclude that $R^{d}=L \oplus K$ (since $H$ is non-onesided), and, consequently, the vector system $H$ is decomposable (we recall that $0<\operatorname{dim} L<d$ ). This completes the proof. 
Lemma 3. Let $H$ be a 2-system, and let $a_{0}, a_{1}, a_{2} \in H$ be minimally dependent vectors. Then each unit vector $v$ of the subspace $L=\operatorname{lin}\left(a_{0}, a_{1}, a_{2}\right)$, except maybe $a_{0}, a_{1}, a_{2}$, is a nonparticular vector with respect to the vector system $H \cap L$.

This assertion is evident.

Lemma 4. Let $H \subset R^{d}$ be an indecomposable 2-system and let $a_{0}, a_{1}, a_{2} \in H$ be three minimally dependent vectors. If $d>2$, then at least one of the vectors $a_{0}, a_{1}$, $a_{2}$ is particular with respect to the vector system $H \cap \operatorname{lin}\left(a_{0}, a_{1}, a_{2}\right)$. This means that there exists a closed half-plane $P \subset \operatorname{lin}\left(a_{0}, a_{1}, a_{2}\right)$ with boundary line through the origin, such that $H \cap \operatorname{lin}\left(a_{0}, a_{1}, a_{2}\right) \subset\{e\} \cup P$, where $e$ is one of the vectors $a_{0}$, $a_{1}, a_{2}$.

This is an evident consequence of Lemmas 2 and 3.

Lemma 5. Let $H \subset R^{d}$ be a 2-system and let $a_{0}, a_{1}, a_{2} \in H$ be three minimally dependent vectors, none of which is particular with respect to $H$. If $-a_{0} \in H$ and $d \geq 3$, then the system $H$ is decomposable.

Proof. We assume that $H$ is indecomposable and show that this assumption leads to a contradiction. We may suppose that $\mu_{0} a_{0}+\mu_{1} a_{1}+\mu_{2} a_{2}=0$ with positive coefficients $\mu_{0}, \mu_{1}, \mu_{2}$. Since the vector $a_{0}$ is nonparticular, then

$$
a_{0}=\lambda_{1} b_{1}+\cdots+\lambda_{s} b_{s}\left(b_{i} \neq a_{0}, b_{i} \in H, \lambda_{i}>0, s \geq 0\right)
$$

By virtue of Lemma 1 , we may suppose that the vectors $-a_{0}, b_{1}, \ldots, b_{s}$ are minimally dependent. Consequently, $s=2$ (since md $H=2$ ), that is, $a_{0}=\lambda_{1} b_{1}+$ $\lambda_{2} b_{2}$. If now $b_{1}, b_{2} \notin \operatorname{lin}\left(a_{0}, a_{1}, a_{2}\right)$, then the vectors $a_{0}, a_{1}, b_{1}$ are linearly dependent, and, moreover, the vectors $a_{1}, a_{2}, b_{1}, b_{2}$ are positively dependent (since $\left.a_{0}=-\left(\mu_{1} / \mu_{0}\right) a_{1}-\left(\mu_{2} / \mu_{0}\right) a_{2}=\lambda_{1} b_{1}+\lambda_{2} b_{2}\right)$. This means that md $H \geq 3$ contradicting the condition of the lemma. Consequently, $b_{1}, b_{2}$ belong to the subspace $L=\operatorname{lin}\left(a_{0}, a_{1}, a_{2}\right)$, that is, $a_{0}$ is a nonparticular vector with respect to the system $H \cap L$. Therefore, by virtue of Lemma 4 (and according to our assumption about the indecomposability of $H$ ), one of the vectors $a_{1}, a_{2}$ (say $a_{1}$ ) is particular with respect to the vector system $H \cap L$. This means that $H \cap L \subset\left\{a_{1}\right\} \cup P$, where $P \subset L$ is a closed half-plane with boundary line through the origin, such that $a_{0},-a_{0}, a_{2} \in P$. Now from the equality $a_{0}=\lambda_{1} b_{1}+\lambda_{2} b_{2}$ (where $\lambda_{1}>0, \lambda_{2}>0$, $\left.b_{1}, b_{2} \in H \cap L\right)$ we deduce that $b_{1}=a_{1}$ and $b_{0} \in$ relint $P, b_{2} \neq a_{2}$. Moreover, the vectors $a_{1}, a_{2}, b_{2}$ are minimally dependent.

Since $a_{1}$ is a nonparticular vector with respect to $H$, then $a_{1}=v_{1} c_{1}+\cdots+v_{t} c_{t}$ $\left(c_{i} \neq a_{1}, c_{i} \in H, v_{i}>0 ; t \geq 2\right)$. Applying Lemma 1 to the vectors $-a_{1}, c_{1}, \ldots, c_{t}$, we may suppose that the vectors $c_{1}, \ldots, c_{t}$ are linearly independent. Let us denote by $l_{1}$ the line through the origin that contains $a_{1}$. The equality $a_{1}=v_{1} c_{1}+\cdots+$ $v_{t} c_{t}$ implies $l_{1} \subset \operatorname{lin}\left(c_{1}, \ldots, c_{t}\right)$. So the following two cases are possible: (a) $L \cap \operatorname{lin}\left(c_{1}, \ldots, c_{t}\right)=l_{1}$ and (b) $L \subset \operatorname{lin}\left(c_{1}, \ldots, c_{t}\right)$. We consider these two cases separately. 
(a) Since the vectors $a_{1}, a_{2}, b_{2}$ are minimally dependent, then $a_{2} \notin l_{1}$. This means that $a_{2} \in L \sim \operatorname{lin}\left(c_{1}, \ldots, c_{t}\right)$, and, therefore, $a_{2} \notin \operatorname{lin}\left(c_{1}, \ldots, c_{t}\right)$. Consequently, the vectors $a_{2}, c_{1}, \ldots, c_{t}$ are linearly independent. On the other hand, there exist positive numbers $\alpha, \beta$, such that $a_{1}+\alpha a_{2}+\beta b_{2}=0$ (since $a_{1}, a_{2}, b_{2}$ are minimally dependent), and, hence, $-\alpha a_{2}-\beta b_{2}=v_{1} c_{1}+\cdots+v_{t} c_{t}$. We have obtained $t+2 \geq 4$ minimally dependent vectors $a_{2}, b_{2}, c_{1}, \ldots, c_{t} \in H$, contradicting the condition md $H=2$. So, case (a) leads to a contradiction.

(b) Let us denote by $K$ the set of all vectors $x=\gamma_{1} c_{1}+\cdots+\gamma_{1} c_{t}$ with nonnegative coefficients $\gamma_{1}, \ldots, \gamma_{t}$. The set $K$ is a closed convex cone with apex at the origin. By virtue of the relation $a_{1}=v_{1} c_{1}+\cdots+v_{t} c_{t}$, with positive $v_{1}, \ldots, v_{t}$, we have $a_{1} \in$ relint $K$. Furthermore, since the vectors $c_{1}, \ldots, c_{t}$ are linearly independent, then the cone $K$ is pointed (that is, it does not contain any line). Consequently, $K \cap L$ is a convex angle distinct from a half-plane, and $a_{1} \in \operatorname{relint}(K \cap L)$. Since $K \cap L$ is not a half-plane, then at least one of the vectors $a_{0},-a_{0}$ does not belong to $K \cap L$; let, for example, $a_{0} \notin K \cap L$. This means that one side of the convex angle $K \cap L$ has with the closed half-plane $P$ the only common point 0 and is situated between the vectors $a_{0}, a_{1}$. So, denoting by $q$ the unit vector directed along this side, we conclude that $-q=\sigma b_{2}+\tau\left(-a_{0}\right)$ with positive $\sigma, \tau$. Furthermore, since $q \in$ relbd $K$ then we may choose from $c_{1}, \ldots, c_{t}$ several vectors, say $c_{1}, \ldots, c_{p}(p<t)$, such that $q=\rho_{1} c_{1}+\cdots+\rho_{p} c_{p}$ with positive $\rho_{1}, \ldots, \rho_{p}$ and $L$ is not contained in $\operatorname{lin}\left(c_{1}, \ldots, c_{p}\right)$. Consequently, $\sigma b_{2}+\tau\left(-a_{0}\right)+$ $\rho_{1} c_{1}+\cdots+\rho_{p} c_{p}=0$. Moreover, the vectors $c_{1}, \ldots, c_{p}$ are linearly independent and $a_{0} \notin \operatorname{lin}\left(c_{1}, \ldots, c_{p}\right)$, and, consequently, $a_{0}, c_{1}, \ldots, c_{p}$ are linearly independent. Thus, the vectors $b_{2},-a_{0}, c_{1}, \ldots, c_{p}$ are minimally dependent, and, hence, md $H \geq p+1 \geq 3$, which is false. So, case (b) leads to a contradiction, too.

We see that the assumption about the indecomposability of $H$ leads to a contradiction. This completes the proof.

Lemma 6. Let $H \subset R^{d}$ be an indecomposable 2-system, and let $a_{0}, a_{1}, a_{2} \in H$ be minimally dependent vectors, none of which is particular with respect to $H$. Then there exist vectors $b_{1}, b_{2}$, each of which is distinct from $a_{2}$, such that the vectors $a_{0}$, $b_{1}, b_{2}$ are minimally dependent.

Proof. We may suppose that $\mu_{0} a_{0}+\mu_{1} a_{1}+\mu_{2} a_{2}=0$ with positive coefficients. Since the vector $a_{2}$ is not particular, then $a_{2}=\lambda_{1} c_{1}+\cdots+\lambda_{k} c_{k}$, where $c_{1}, \ldots$, $c_{k} \in H, c_{i} \neq a_{2}, \lambda_{i}>0$. Consequently,

$$
\frac{\mu_{0}}{\mu_{2}} a_{0}+\frac{\mu_{1}}{\mu_{2}} a_{1}+\lambda_{1} c_{1}+\cdots+\lambda_{k} c_{k}=0 .
$$

We may suppose that each of the vectors $c_{1}, \ldots, c_{k}$ is distinct from $a_{0}$ and $a_{1}$. Let us choose from $a_{1}, c_{1}, \ldots, c_{k}$ vectors, such that $a_{0}$ and the chosen vectors are minimally dependent (see Lemma 1). It is clear that this minimally dependent system is a triplet $a_{0}, b_{1}, b_{2}$ (because $-a_{0} \notin H$, see Lemma 5), and each of the vectors $b_{1}, b_{2}$ is distinct from $a_{2}$ (but it is possible that one of the vectors $b_{1}, b_{2}$ coincides with $a_{1}$ ). 
Lemma 7. Let $H \subset R^{d}$ be an indecomposable 2-system and let $a_{0}, a_{1}, a_{2} \in H$ be minimally dependent vectors, none of which is particular with respect to $H$. If $d>2$, then the intersection $H \cap \operatorname{lin}\left(a_{0}, a_{1}, a_{2}\right)$ consists only of the vectors $a_{0}, a_{1}, a_{2}$.

Proof. According to Lemma 4, there exists a vector among $a_{0}, a_{1}, a_{2}$, say $a_{0}$, that is particular with respect to the vector system $H \cap \operatorname{lin}\left(a_{0}, a_{1}, a_{2}\right)$. This means that $H \cap \operatorname{lin}\left(a_{0}, a_{1}, a_{2}\right) \subset\left\{a_{0}\right\} \cup P_{0}$, where $P_{0}$ is a closed half-plane with boundary line through the origin. Let us suppose that there exists a vector $e \in H \cap \operatorname{lin}\left(a_{0}, a_{1}, a_{2}\right)$ that does not belong to $\left\{a_{0}, a_{1}, a_{2}\right\}$. Then, according to Lemma $5,-e$ does not coincide with any vector $a_{0}, a_{1}, a_{2}$. Consequently, $-e$ is a positive linear combination of two of the vectors $a_{0}, a_{1}, a_{2}$, say, $-e=\lambda_{0} a_{0}+$ $\lambda_{1} a_{1}$, where $\lambda_{0}>0, \lambda_{1}>0$. This means that the vectors $e, a_{0}, a_{1}$ are minimally dependent. By virtue of Lemma 6 there exist vectors $b_{1}, b_{2}$, each of which is distinct from $a_{0}$, such that $e, b_{1}, b_{2}$ are minimally dependent, that is, $-e=\mu_{1} b_{1}+\mu_{2} b_{2}$ with positive coefficients $\mu_{1}, \mu_{2}$. It is evident that the vectors $b_{1}, b_{2}$ did not belong to $\operatorname{lin}\left(a_{0}, a_{1}, a_{2}\right)$, since otherwise we should have $e, b_{1}, b_{2} \in P_{0}$, contradicting the minimal dependence of these vectors. On the other hand, $e$ is a positive linear combination of some two vectors from $a_{0}, a_{1}, a_{2}$ (since $e$ coincides with no vector $\left.a_{0}, a_{1}, a_{2}\right)$, say, $e=v_{0} a_{0}+v_{2} a_{2}$. So we have $v_{0} a_{0}+v_{2} a_{2}+\mu_{1} b_{1}+\mu_{2} b_{2}=0$. Moreover, the vectors $a_{0}, a_{2}, b_{1}$ are linearly independent, since $b_{1} \notin \operatorname{lin}\left(a_{0}, a_{1}, a_{2}\right)$. Therefore, the vectors $a_{0}, a_{2}, b_{1}, b_{2} \in H$ are minimally dependent, contradicting the condition md $H=2$. This completes the proof.

Lemma 8. Let $H \subset S^{d-1}$ be a 2-system and let $a_{0}, a_{1}, a_{2}$ be three minimally dependent vectors, none of which is particular with respect to $H$. If $d>2$, then there exist vectors $b_{1}, b_{2}$ not belonging to $\operatorname{lin}\left(a_{0}, a_{1}, a_{2}\right)$, such that $a_{0}, b_{1}, b_{2}$ are minimally dependent.

This is an evident consequence of Lemmas 6 and 7.

Lemma 9. Let $H \subset R^{d}$ be a maximal 2-system and let $H^{\Delta}$ be the set of all its particular vectors. If $H^{\Delta} \neq \varnothing$, then for every vector $e \in H^{\Delta}$ we have $-e \in H$.

Proof. We must prove that $\operatorname{md}(H \cup\{-e\})=2$ (it would follow from this that $-e \in H$, since $H$ is maximal). Indeed, let $\alpha(-e)+\gamma_{1} c_{1}+\cdots+\gamma_{m} c_{m}=0$ be a minimal dependence, $c_{1}, \ldots, c_{m} \in H$. Since $e$ is a particular vector, then there exists a hyperplane $N$ through the origin, such that $H \sim\{e\} \subset P$, where $P$ is one of the closed half-spaces determined by $N$. Besides that, we have $e \notin P$, and, consequently, $-e \in$ int $P$. So, if each of the vectors $c_{1}, \ldots, c_{m}$ is distinct from $e$, then all the vectors $c_{1}, \ldots, c_{m}$ belong to $H$, and $-e \in$ int $P$, contradicting the minimal dependence of these vectors. This contradiction shows that one of the vectors $c_{1}, \ldots, c_{m}$ coincides with $e$, say $c_{1}=e$. However, then we have $m=1$, and the minimal dependence has the form $e+(-e)=0$. Thus if we have a minimal dependence $\beta_{0} b_{0}+\cdots+\beta_{s} b_{s}=0$ (where $b_{0}, \ldots, b_{s} \in H \cup\{-e\}$ ), then either $-e \notin\left\{b_{0}, \ldots, b_{s}\right\}$, that is, $b_{0}, \ldots, b_{s} \in H$, and then $s \leq 2$, or $-e \in\left\{b_{0}, \ldots, b_{s}\right\}$ and then $s=1$. In any case $s \leq 2$, that is, $\operatorname{md}(H \cup\{-e\})=2$. 
Lemma 10. Let $H \subset R^{d}$ be a maximal 2-system and let $H^{\Delta}$ be the set of all its particular vectors. If $H^{\Delta} \neq \varnothing$, then the vector system $H \cap$ lin $H^{\Delta}$ is non-one-sided in $\operatorname{lin} H^{\Delta}$.

This follows immediately from Lemma 9.

Lemma 11. Let $H \subset R^{d}$ be an indecomposable maximal 2-system and let $H^{\Delta}=$ $\left\{e_{1}, \ldots, e_{k}\right\}$ be the set of all its particular vectors. Then the vectors $e_{1}, \ldots, e_{k}$ are linearly independent.

Proof. Let us suppose that the vectors $e_{1}, \ldots, e_{k}$ are linearly dependent. Without loss of generality we may suppose that a linear dependence has a form $\lambda_{1} e_{1}+\cdots+\lambda_{q} e_{q}=0$, where $e_{1}, \ldots, e_{q-1}$ are linearly independent, $q \leq k, \lambda_{i}>0$ for $i \leq s$ and $\lambda_{i}<0$ for $s<i \leq q$. Then we have

$$
\lambda_{1} e_{1}+\cdots+\lambda_{s} e_{s}+\left|\lambda_{s+1}\right|\left(-e_{s+1}\right)+\cdots+\left|\lambda_{q}\right|\left(-e_{q}\right)=0
$$

that is, the vectors $e_{1}, \ldots, e_{s},-e_{s+1}, \ldots,-e_{q} \in H$ (see Lemma 7) are minimally dependent. Consequently, $q \leq 3$. So we have two cases: (i) $q=2$ and (ii) $q=3$. In case (i) the linear dependence has the form $e_{1}+e_{2}=0$, that is, $e_{2}=-e_{1}$ where $e_{1}, e_{2} \in H^{\Delta}$. This means that $H \sim\left\{e_{1} \cup e_{2}\right\} \subset P_{1} \cap P_{2}, e_{1} \notin P_{1}, e_{2} \notin P_{2}$ where $P_{1}$, $P_{2}$ are closed half-spaces with boundary hyperplanes through the origin. If now bd $P_{1}=$ bd $P_{2}$, then $H \subset \operatorname{lin}\left\{e_{1}\right\} \oplus$ bd $P_{1}$, contradicting the indecomposability of $H$. If bd $P_{1} \neq$ bd $P_{2}$, then it is easily shown that $P_{1} \cap P_{2} \cup\left\{e_{1} \cup e_{2}\right\}$ is contained in a half-space with boundary hyperplane through the origin, contradicting the non-one-sideness of $H$. In case (ii) the linear dependence has the form $\lambda_{1} e_{1}+$ $\lambda_{2} e_{2}+\lambda_{3} e_{3}=0$. This means, according to Lemma 9, that the vectors $e_{1}, e_{2}, e_{3}$, $-e_{1},-e_{2},-e_{3}$ belong to the two-dimensional plane lin $\left(e_{1}, e_{2}, e_{3}\right)$. Then each vector $v \in \operatorname{lin}\left(e_{1}, e_{2}, e_{3}\right)$ is nonparticular with respect to the vector system $H \cap \operatorname{lin}\left(e_{1}, e_{2}, e_{3}\right)$, contradicting the indecomposability of the system $H$ (see Lemma 2). This completes the proof.

\section{Proof of Theorem 8}

Let $H \subset S^{d-1}$ be a maximal, indecomposable 2-system without particular vectors; $d \geq 3$. Let, furthermore, $a_{0}, a_{1}, a_{2}$ be three minimally dependent vectors. According to Lemma 8 there exist vectors $b_{0}, b_{1}, b_{2}, c_{0}, c_{1}, c_{2}$ not belonging to $\operatorname{lin}\left(a_{0}, a_{1}, a_{2}\right)$, such that the triplets $\left(a_{0}, b_{0}, c_{0}\right),\left(a_{1}, b_{1}, c_{1}\right),\left(a_{2}, b_{2}, c_{2}\right)$ are minimally dependent. Then the spaces

$L_{0}=\operatorname{lin}\left(a_{0}, a_{1}, a_{2}, b_{0}, c_{0}\right), \quad L_{1}=\operatorname{lin}\left(a_{0}, a_{1}, a_{2}, b_{1}, c_{1}\right), \quad L_{2}=\operatorname{lin}\left(a_{0}, a_{1}, a_{2}, b_{2}, c_{2}\right)$

are three-dimensional. First we prove that these three subspaces are pairwise distinct (and, consequently, $d \geq 4$ ). 
Let us suppose, counterwise, that two of these subspaces coincide, say $L_{1}=L_{2}$. Then the vectors $a_{1}, a_{2}, b_{1}$ form a basis in the three-dimensional space $L_{1}=L_{2}$. Without loss of generality we may suppose that $b_{1}, b_{2}$ lie in one open half-space with boundary $\operatorname{lin}\left(a_{0}, a_{1}, a_{2}\right)$, and $c_{1}, c_{2}$ lie in the other open half-space. Since $a_{1}, b_{1}, c_{1}$ are minimally dependent, then

$$
b_{1}=-\alpha_{1} a_{1}-\gamma_{1} c_{1} \quad\left(\alpha_{1}>0, \gamma_{1}>0\right)
$$

Similarly,

$$
b_{2}=-\alpha_{2} a_{2}-\gamma_{2} c_{2} \quad\left(\alpha_{2}>0, \gamma_{2}>0\right) \text {. }
$$

Furthermore, since $b_{1}, b_{2}$ lie in one open half-space with boundary $\operatorname{lin}\left(a_{0}, a_{1}, a_{2}\right)$, we have

$$
\lambda b_{2}=b_{1}+\mu a_{1}+v a_{2} \quad(\lambda>0) .
$$

Without loss of generality we may suppose (changing the roles of the indices 1,2 , if necessary) that $\mu \geq 0$. Now we obtain, from the relations (7) and (8),

$$
\lambda \gamma_{2} c_{2}+b_{1}+\mu a_{1}+\left(v+\lambda \alpha_{2}\right) a_{2}=0 \quad\left(\lambda>0, \mu \geq 0, \gamma_{2}>0\right) .
$$

Besides that, we have a positive dependence

$$
p_{0} a_{0}+p_{1} a_{1}+a_{2}=0
$$

(since $a_{0}, a_{1}, a_{2}$ are minimally dependent).

If the coefficient $v+\lambda \alpha_{2}$ is negative, that is, $v+\lambda \alpha_{2}=-\sigma(\sigma>0)$, then we obtain, from (9) and (10),

$$
\lambda \gamma_{2} c_{2}+b_{1}+\sigma p_{0} a_{0}+\left(\sigma p_{1}+\mu\right) a_{1}=0 .
$$

This is a minimal dependence (since all the coefficients are positive, and $a_{0}, a_{1}, b_{1}$ are linearly independent), contradicting the equality $\mathrm{md} H=2$. Consequently, $v+\lambda \alpha_{2} \geq 0$.

Furthermore, if both the coefficients $\mu, v+\lambda \alpha_{2}$ in (9) are positive, then (9) is a minimal dependence, contradicting the equality md $H=2$. So both the coefficients $\mu, v+\lambda \alpha_{2}$ are nonnegative, and at least one of them is equal to 0 . However, the case $\mu=v+\lambda \alpha_{2}=0$ is impossible. Indeed, in this case we would have $\lambda \gamma_{2} c_{2}+b_{1}=0$ and, consequently, $c_{2}+b_{1}=0$ (since $\left|c_{2}\right|=\left|b_{1}\right|=1$ ), that is, $-b_{1}=c_{2} \in H$, contradicting Lemma 5 .

Thus, the following two cases are possible:

$$
\begin{aligned}
\lambda \gamma_{2} c_{2}+b_{1}+\mu a_{1} & =0 & & \left(\lambda \gamma_{2}>0, \mu>0\right), \\
\text { (b) } & \lambda \gamma_{2} c_{2}+b_{1}+\left(v+\lambda \alpha_{2}\right) a_{2} & =0 & \left(\lambda \gamma_{2}>0, v+\lambda \alpha_{2}>0\right) .
\end{aligned}
$$


In the case of (a) the vectors $c_{2}, b_{1}, a_{1}$ are minimally dependent, and by virtue of Lemma 7 we have $c_{2}=c_{1}$. In case (b) the vectors $c_{2}, b_{1}, a_{2}$ are minimally dependent, and by virtue of Lemma 7 we have $b_{1}=b_{2}$. Uniting the two cases (a) and (b), we obtain that in any case there are among $b_{1}, b_{2}, c_{1}, c_{2}$ only three distinct vectors. Without loss of generality we can suppose that $b_{1}=b_{2}$, that is, we have three distinct vectors $b_{1}=b_{2}, c_{1}, c_{2}$.

Now let us consider the third three-dimensional space $L_{0}$. Since $a_{0}, b_{0}, c_{0}$ are minimally dependent, then we have

$$
b_{0}=-\alpha_{0} a_{0}-\gamma_{0} c_{0} \quad\left(\alpha_{0}>0, \gamma_{0}>0\right)
$$

Consequently (by virtue of relation (10)),

$$
\begin{gathered}
p_{0} \alpha_{1} \alpha_{2}\left(b_{0}+\gamma_{0} c_{0}\right)+p_{1} \alpha_{0} \alpha_{2}\left(b_{1}+\gamma_{1} c_{1}\right)+p_{2} \alpha_{0} \alpha_{1}\left(b_{2}+\gamma_{2} c_{2}\right) \\
=-\alpha_{0} \alpha_{1} \alpha_{2}\left(p_{0} a_{0}+p_{1} a_{1}+p_{2} a_{2}\right)=0 .
\end{gathered}
$$

This is a positive dependence among the vectors $b_{0}, b_{1}, c_{0}, c_{1}, c_{2}$ (since $b_{2}=b_{1}$ ). If now $b_{0} \notin L_{1}$, then the vectors $b_{1}, c_{1}, c_{2}, b_{0}$ are linearly independent, and, according to (12), the vectors $b_{0}, c_{0}, b_{1}, c_{1}, c_{2}$ are minimally dependent, contradicting the equality md $H=2$. Hence, $b_{0} \in L_{1}$, that is, $L_{0}=L_{1}=L_{2}$.

Thus, from the equality $L_{1}=L_{2}$ we have deduced that there are among $b_{1}, b_{2}$, $c_{1}, c_{2}$ only three distinct vectors; say, $b_{1}=b_{2}$. Furthermore, from the equality $L_{0}=L_{1}$ we obtain, similarly, that there are among $b_{0}, b_{1}, c_{0}, c_{1}$ only three distinct vectors. So we have two possibilities: (i) $b_{0}=b_{1}$ and (ii) $c_{0}=c_{1}$. In case (ii) we have $b_{1}=b_{2}, c_{1} \neq c_{2}$ and $c_{0}=c_{1}, b_{0} \neq b_{1}$. This means that $b_{0} \neq b_{2}, c_{0} \neq c_{2}$, contradicting the equality $L_{0}=L_{2}$. Consequently, case (i) must hold, that is, $b_{0}=b_{1}=b_{2}$, and $c_{0}, c_{1}, c_{2}$ are pairwise different. In this case we have (by virtue of (12))

$$
p_{0} \alpha_{1} \alpha_{2}\left(b_{0}+\gamma_{0} c_{0}\right)+p_{1} \alpha_{0} \alpha_{2}\left(b_{0}+\gamma_{1} c_{1}\right)+p_{2} \alpha_{0} \alpha_{1}\left(b_{0}+\gamma_{2} c_{2}\right)=0
$$

that is, the vectors $b_{0}, c_{0}, c_{1}, c_{2}$ are positively dependent. Besides that, the vectors $b_{0}, c_{0}, c_{1}$ are linearly independent. Consequently, the vectors $b_{0}, c_{0}, c_{1}, c_{2}$ are minimally dependent, contradicting the equality md $H=2$. The contradiction obtained shows that all the three-dimensional spaces $L_{0}, L_{1}, L_{2}$ are pairwise different.

Let us denote by $Q$ the four-dimensional space $\operatorname{lin}\left(a_{0}, a_{1}, a_{2}, b_{1}, b_{2}, c_{1}, c_{2}\right)$. If $b_{0} \notin Q$, then the vectors $b_{1}, b_{2}, c_{1}, c_{2}, b_{0}$ are linearly independent. Consequently, the vectors $b_{0}, b_{1}, b_{2}, c_{0}, c_{1}, c_{2}$ are minimally dependent, contradicting the equality md $H=2$. This shows that all the vectors $a_{0}, a_{1}, a_{2}, b_{0}, b_{1}, b_{2}, c_{0}, c_{1}, c_{2}$ lie in the four-dimensional space $Q$.

Let us now consider two triplets $\left(a_{1}, b_{1}, c_{1}\right)$ and $\left(a_{2}, b_{2}, c_{2}\right)$. We have $b_{2} \notin \operatorname{lin}\left(a_{1}, a_{2}, b_{1}\right)=L_{1}$ (since $\left.L_{1} \neq L_{2}\right)$. Consequently, $a_{1}, a_{2}, b_{1}, b_{2}$ is a basis in $Q$. Now we have $-b_{0}=p_{1}+p_{2}$, where $p_{1} \in \operatorname{lin}\left(a_{1}, b_{1}\right), p_{2} \in \operatorname{lin}\left(a_{2}, b_{2}\right)$. It is clear that $p_{1} \neq 0, p_{2} \neq 0$ (since $b_{0} \notin \operatorname{lin}\left(a_{1}, b_{1}, c_{1}\right), b_{0} \notin \operatorname{lin}\left(a_{2}, b_{2}, c_{2}\right)$ ). Furthermore, if $p_{1}$ coincides with no vector $a_{1}, b_{1}, c_{1}$ (up to a positive coefficient), then $p_{1}$ can be 
written as a positive linear combination of two of the vectors $a_{1}, b_{1}, c_{1}$, say, $p_{1}=\beta b_{1}+\gamma c_{1}$. Hence, $b_{0}+\beta b_{1}+\gamma c_{1}=-p_{2} \in \operatorname{lin}\left(a_{2}, b_{2}, c_{2}\right)$. Now, $-p_{2} \neq 0$ is a positive linear combination of one or two vectors $a_{2}, b_{2}, c_{2}$, that is, we obtain four or five minimally dependent vectors $b_{0}, b_{1}, c_{1}, \ldots$, contradicting the equality md $H=2$. Thus, $p_{1}$ must coincide (up to a positive coefficient) with one of the vectors $a_{1}, b_{1}, c_{1}$. Similarly, $p_{2}$ must coincide with one of the vectors $a_{2}, b_{2}, c_{2}$. The same reasoning shows that $-c_{0}=q_{1}+q_{2}$, where $q_{1}$ coincides with one of the vectors $a_{1}, b_{1}, c_{1}$, and $q_{2}$ coincides with one of the vectors $a_{2}, b_{2}, c_{2}$.

Let us show that $p_{1} \neq a_{1}$. Indeed, in the case $p_{1}=a_{1}, p_{2}=a_{2}$ we obtain $b_{0}=-p_{1}-p_{2} \in \operatorname{lin}\left(a_{1}, a_{2}\right)=\operatorname{lin}\left(a_{0}, a_{1}, a_{2}\right)$, which is impossible by virtue of Lemma 7 (we recall that the nine vectors $a_{0}, a_{1}, a_{2}, b_{0}, b_{1}, b_{2}, c_{0}, c_{1}, c_{2}$ are pairwise different). Furthermore, in the case $p_{1}=a_{1}, p_{2}=b_{2}$ we conclude that $b_{0}, a_{1}, b_{2}$ are linearly dependent, and, consequently, $a_{1} \in \operatorname{lin}\left(b_{0}, b_{2}\right)=\operatorname{lin}\left(b_{0}, b_{1}, b_{2}\right)$, which is impossible. Finally, in the case $p_{1}=a_{1}, p_{2}=c_{2}$ we find that the vectors $b_{0}, a_{1}, c_{2}$ are linearly dependent, and, consequently, the vectors $b_{0}, a_{1}, c_{2}, a_{2}$ lie in one three-dimensional space. This means that $\operatorname{lin}\left(a_{1}, a_{2}, b_{0}\right)=\operatorname{lin}\left(a_{1}, a_{2}, c_{2}\right)$, that is, $L_{0}=L_{2}$, contradicting what was proved above. Thus, $p_{1} \neq a_{1}$ (up to a positive coefficient). Similarly, $p_{2} \neq a_{2}, q_{1} \neq a_{1}, q_{2} \neq a_{2}$.

We see that $p_{1}$ is equal to either $b_{1}$ or $c_{1}$, and $p_{2}$ is equal to either $b_{2}$ or $c_{2}$ (up to a positive coefficient). Let us now remark that (by virtue of pairwise distinctness of the spaces $L_{0}, L_{1}, L_{2}$ ) we may interchange the symbols $b_{1}$ and $c_{1}$; similarly, we may interchange the symbols $b_{2}$ and $c_{2}$. Therefore, we may suppose that $p_{1}=\lambda_{1} b_{1}$, $p_{2}=\lambda_{2} b_{2}\left(\lambda_{1}>0, \lambda_{2}>0\right)$, that is, the three vectors $b_{0}=-p_{1}-p_{2}, b_{1}, b_{2}$ are minimally dependent.

Now we conclude that $q_{1}$ is equal to either $b_{1}$ or $c_{1}$ and $q_{2}$ is equal to either $b_{2}$ or $c_{2}$, but neither $q_{1}=b_{1}$ nor $q_{2}=b_{2}$ is valid. For, if $q_{1}$ was equal to $b_{1}$, then the vectors $b_{0}, c_{0}$ (and consequently $a_{0}, a_{2}$ ) would lie in one three-dimensional space $\operatorname{lin}\left(b_{1}, b_{2}, c_{2}\right)$, that is, $L_{0}=L_{2}$, contradicting what was previously proved. Consequently, $q_{1}=\mu_{1} c_{1}$. Similarly, $q_{2}=\mu_{2} c_{2}$. This means that the three vectors $c_{0}=-q_{1}-q_{2}, c_{1}, c_{2}$ are minimally dependent.

We may sum up. The vectors $a_{1}, b_{1}, a_{2}, b_{2}$ form a basis in $Q$. Moreover, each triplet $\left(a_{0}, a_{1}, a_{2}\right),\left(b_{0}, b_{1}, b_{2}\right),\left(c_{0}, c_{1}, c_{2}\right),\left(a_{0}, b_{0}, c_{0}\right),\left(a_{1}, b_{1}, c_{1}\right),\left(a_{2}, b_{2}, c_{2}\right)$ is minimally dependent, that is, we have positive dependences:

$$
\begin{array}{rrr}
a_{0}+\lambda_{1} a_{1}+\lambda_{2} a_{2}=0, & b_{0}+\mu_{1} b_{1}+\mu_{2} c_{2}=0, & p a_{0}+p^{\prime} b_{0}+c_{0}=0, \\
c_{0}+v_{1} c_{1}+v_{2} c_{2}=0, & q a_{1}+q^{\prime} b_{1}+c_{1}=0, & \tau a_{2}+\tau^{\prime} b_{2}+c_{2}=0 .
\end{array}
$$

We now fix the basis $e_{1}, e_{2}, e_{3}, e_{4}$ in $Q$ by the relations

$$
e_{1}=p \lambda_{1} a_{1}, \quad e_{2}=p \lambda_{2} a_{2}, \quad e_{3}=p^{\prime} \mu_{1} b_{1}, \quad e_{4}=p^{\prime} \mu_{2} b_{2}
$$

Then, by virtue of (13),

$$
a_{0}=-\frac{1}{p}\left(e_{1}+e_{2}\right), \quad b_{0}=-\frac{1}{p^{\prime}}\left(e_{3}+e_{4}\right), \quad c_{0}=e_{1}+e_{2}+e_{3}+e_{4} .
$$


Furthermore, according to (14), we have

$$
c_{0}=v_{1}\left(q a_{1}+q^{\prime} b_{1}\right)+v_{2}\left(\tau a_{2}+\tau^{\prime} b_{2}\right)=\frac{v_{1} q}{p \lambda_{1}} e_{1}+\frac{v_{1} q^{\prime}}{p^{\prime} \mu_{1}} e_{3}+\frac{v_{2} \tau}{p \lambda_{2}} e_{2}+\frac{v_{2} \tau^{\prime}}{p^{\prime} \mu_{2}} e_{4} .
$$

Consequently,

$$
\frac{v_{1} q}{p \lambda_{1}}=1, \quad \frac{v_{1} q^{\prime}}{p^{\prime} \mu_{1}}=1, \quad \frac{v_{2} \tau}{p \lambda_{2}}=1, \quad \frac{v_{2} \tau^{\prime}}{p^{\prime} \mu_{2}}=1
$$

According to these relations, the two last equalities in (14) take the form

$$
\frac{p \lambda_{1}}{v_{1}} a_{1}+\frac{p^{\prime} \mu_{1}}{v_{1}} b_{1}+c_{1}=0, \quad \frac{p \lambda_{2}}{v_{2}} a_{2}+\frac{p^{\prime} \mu_{2}}{v_{2}} b_{2}+c_{2}=0
$$

that is,

$$
c_{1}=-\frac{1}{v_{1}}\left(e_{1}+e_{3}\right), \quad c_{2}=-\frac{1}{v_{2}}\left(e_{2}+e_{4}\right)
$$

By virtue of (15)-(17) (and taking into account that $\left|a_{i}\right|=\left|b_{i}\right|=\left|c_{i}\right|=1$, since $H \subset S^{d-1}$ ) we obtain $\left\{a_{0}, a_{1}, a_{2}, b_{0}, b_{1}, b_{2}, c_{0}, c_{1}, c_{2}\right\}=\operatorname{norm}\left\{-e_{1}-e_{2}, e_{1}, e_{2}\right.$, $\left.-e_{3}-e_{4}, e_{3}, e_{4}, e_{1}+e_{2}+e_{3}+e_{4},-e_{1}-e_{3},-e_{2}-e_{4}\right\}=H^{(0)}$ (see the statement of Theorem 8). Consequently, $H \supset H^{(0)}$ (because $a_{i}, b_{i}, c_{i} \in H$ ).

It remains to prove that $d=4$, and $H$ does not contain vectors except the vectors belonging to $H^{(0)}$. For this purpose we remark first that each vector $v \in Q$ is nonparticular with respect to vector system $H^{(0)}$. Indeed, $H^{(0)}$ is non-one-sided in the space $Q$ (even the vector system $e_{1}, e_{2},-e_{1}-e_{2} ; e_{3}, e_{4},-e_{3}-e_{4}$ is not one-sided). Furthermore, if $v \notin H^{(0)}$, then $v$ is nonparticular (since $H^{(0)}$ is non-onesided). Finally, if $v \in H^{(0)}$, then $v$ is nonparticular too, since

$$
e_{1}=\left(e_{1}+e_{2}+e_{3}+e_{4}\right)+e_{4}+\left(-e_{2}-e_{4}\right)+\left(-e_{3}-e_{4}\right)
$$

(similarly for $e_{2}, e_{3}, e_{4}$ ),

$$
-e_{1}-e_{2}=\left(-e_{1}-e_{3}\right)+\left(-e_{2}-e_{4}\right)+e_{3}+e_{4}
$$

(similarly for $-e_{1}-e_{3},-e_{2}-e_{3},-e_{2}-e_{4}$ ), and $e_{1}, e_{2}, e_{3}$ is, evidently, nonparticular, too. Now Lemma 2 shows that $d=4$ (i.e., $R^{d}=Q$ ), since the vector system $H$ is indecomposable.

It remains to prove that the 2 -system coincides with $H^{(0)}$. Indeed, let $v \in H$. If $v \in \operatorname{lin}\left(e_{1}, e_{3}\right)$, then either $v=e_{1}$, or $v=e_{3}$, or $v=-e_{1}-e_{3}$ (see Lemma 7), that is, $v \in H^{(0)}$. Similarly, if $v \in \operatorname{lin}\left(e_{2}, e_{4}\right)$, then $v \in H^{(0)}$. Let, finally, $v \in H \sim \operatorname{lin}\left(e_{1}, e_{3}\right) \sim$ $\operatorname{lin}\left(e_{2}, e_{4}\right)$. Then $v=v^{*}+v^{* *}$ where $v^{*} \in \operatorname{lin}\left(e_{1}, e_{3}\right), v^{* *} \in \operatorname{lin}\left(e_{2}, e_{4}\right)$ and $v^{*} \neq 0$, $v^{* *} \neq 0$. The vector $-v^{*}$ is representable as a positive linear combination of one or two vectors $e_{1}, e_{3},-e_{1}-e_{3}$. If $-v^{*}$ is expressed by two vectors, say 
$-v^{*}=\lambda e_{1}+\mu\left(-e_{1}-e_{3}\right)(\lambda>0, \mu>0)$, then we have $v+\lambda e_{1}+\mu\left(-e_{1}-e_{3}\right)-$ $v^{* *}=0$, and (representing $-v^{* *}$ as a positive linear combination of one or two vectors $e_{2}, e_{4},-e_{2}-e_{4}$ ) we obtain a minimal dependence between four or five vectors, contradicting the equality md $H=2$. So, $-v^{*}$ coincides with one of the vectors $e_{1}, e_{3},-e_{1}-e_{3}$, and, similarly, $-v^{* *}$ coincides with one of the vectors $e_{2}, e_{4},-e_{2}-e_{4}$ (up to positive coefficients). Thus, we have the following cases $(\alpha, \beta$ are positive):

$$
\begin{aligned}
v=-\alpha e_{1}-\beta e_{2}, \quad v=-\alpha e_{3}-\beta e_{4}, & v=\alpha\left(e_{1}+e_{3}\right)+\beta\left(e_{2}+e_{4}\right) ; \\
v=-\alpha e_{1}-\beta e_{4}, & v=-\alpha e_{3}-\beta e_{2} \\
v=\alpha\left(e_{1}+e_{3}\right)-\beta e_{2}, & v=\alpha\left(e_{1}+e_{3}\right)-\beta e_{4} \\
v=\alpha\left(e_{2}+e_{4}\right)-\beta e_{1}, & v=\alpha\left(e_{2}+e_{4}\right)-\beta e_{3}
\end{aligned}
$$

In the first case of (18) we have

$$
\begin{array}{r}
v+\alpha\left(e_{1}+e_{2}+e_{3}+e_{4}\right)+(\alpha-\beta)\left(-e_{2}-e_{4}\right)+\alpha\left(-e_{3}-e_{4}\right)+(\alpha-\beta) e_{4}=0 \\
(\text { for } \alpha>\beta), \\
v+\beta\left(e_{1}+e_{2}+e_{3}+e_{4}\right)+(\beta-\alpha)\left(-e_{1}-e_{3}\right)+\beta\left(-e_{3}-e_{4}\right)+(\beta-\alpha) e_{3}=0 \\
(\text { for } \alpha<\beta) .
\end{array}
$$

So, if $\alpha \neq \beta$, then $\operatorname{md}\left(H \cup\left\{-\alpha e_{1}-\beta e_{2}\right\}\right) \geq 4$. Consequently, $-\alpha e_{1}-\beta e_{2} \in H$ only in the case $\alpha=\beta=1 /\left|e_{1}+e_{2}\right|$ (since $|v|=1$ ). In other words, if $v=-\alpha e_{1}-$ $\beta e_{2} \in H$, then $v \in H^{(0)}$. The two last cases of (18) may be considered similarly. As to (19) and (20), there exists no positive $\alpha, \beta$, for which $v \in H$ in these cases. For example: if $v=-\alpha e_{1}-\beta e_{4}$, then

$$
v+\alpha\left(e_{1}+e_{2}+e_{3}+e_{4}\right)+\alpha\left(-e_{2}-e_{4}\right)+\alpha\left(-e_{3}-e_{4}\right)+(\alpha+\beta) e_{4}=0
$$

if $v=\alpha\left(e_{1}+e_{3}\right)-\beta e_{2}$, then

$$
v+\alpha\left(-e_{2}-e_{3}\right)+\alpha\left(-e_{1}-e_{4}\right)+\alpha e_{4}+(\alpha+\beta) e_{2}=0
$$

Thus, if $v \in H$, then $v \in H^{(0)}$, that is, $H \subset H^{(0)}$. This completes the proof.

Looking the proof through, we notice that we have used only the fact that if $e_{0}, e_{1}, e_{2} \in H$ are minimally dependent vectors, then none of them is particular. So the following stronger result is true.

Theorem 8'. Let $d \geq 3$ and let $H \subset S^{d-1}$ be an indecomposable 2-system, such that if $e_{0}, e_{1}, e_{2} \in H$ are minimally dependent vectors, then none of them is particular with respect to $H$. Under these conditions, $d=4$ and $H=H^{(0)}$.

This theorem is used in the next section. 


\section{Proof of Theorem 9}

Let $L=\operatorname{lin} H^{\Delta}$ and let $N$ be a complementary subspace for $L$, that is, $R^{d}=L \oplus N$. We denote by $\pi$ the natural projection $R^{d} \rightarrow N$ with kernel $L$. We consider at first the case when $1 \leq \operatorname{dim} L<d$, that is $1 \leq \operatorname{dim} N<d$. Then $H^{*}=$ norm $\pi(H)$ is a vector system in $N$ which is, evidently, non-one-sided. We are going to show that $\mathrm{md} H^{*}=1$. This is obvious if $\operatorname{dim} N=1$. So we must consider the case $\operatorname{dim} N \geq 2$. Let us suppose that $m d H^{*}=m>1$ and let $a_{0}^{*}, a_{1}^{*}, \ldots, a_{m}^{*} \in H^{*}$ be minimally dependent vectors, and $a_{0}, a_{1}, \ldots, a_{m}$ be their representatives, that is, $a_{i} \in \pi^{-1}\left(a_{i}^{*}\right) \cap H(i=0, \ldots, m)$. Let $\lambda_{0} a_{0}^{*}+\cdots+\lambda_{m} a_{m}^{*}=0$ be the corresponding positive dependence. Then the vector $w=\lambda_{0} a_{0}+\cdots+\lambda_{m} a_{m}$ belongs to the subspace $L$. If $w \neq 0$, then

$$
\lambda_{0} a_{0}+\cdots+\lambda_{m} a_{m}+\mu_{1} b_{1}+\cdots+\mu_{k} b_{k}=0
$$

where $b_{1}, \ldots, b_{k} \in L \cap H$ are linearly independent and $\mu_{i}>0(k \geq 1)$. We obtain $m+1+k \geq 4$ minimally dependent vectors of the system $H$, contradicting the relation $\mathrm{md} H=2$. Hence $w=0$, that is, $\lambda_{0} a_{0}+\cdots+\lambda_{m} a_{m}=0$. Consequently, according to relations $m>1$ and $\mathrm{md} H=2$, we have $m=2$, that is, md $H^{*}=2$. So the considered minimal dependence has the form $\lambda_{0} a_{0}^{*}+\lambda_{1} a_{1}^{*}+\lambda_{2} a_{2}^{*}=0$, and, moreover, $\lambda_{0} a_{0}+\lambda_{1} a_{1}+\lambda_{2} a_{2}=0$ for any representatives $a_{i} \in \pi^{-1}\left(a_{i}^{*}\right) \cap H, i=0$, 1, 2. This means that each of the sets $\pi^{-1}\left(a_{i}^{*}\right) \cap H, i=0,1,2$, contains only one element. If now one of the vectors $a_{0}^{*}, a_{1}^{*}, a_{2}^{*}$ (say, $a_{0}^{*}$ ) is particular with respect to the vector system $H^{*}$, then the corresponding vector $a_{0}$ is particular with respect to the vector system $H$, that is, $a_{0} \in H^{\Delta}$. However, this contradicts the relations $H^{\Delta} \subset L, a_{0} \notin L$. Thus if $a_{0}^{*}, a_{1}^{*}, a_{2}^{*} \in H^{*}$ are minimally dependent vectors, then these vectors are nonparticular with respect to $H^{*}$. Now we conclude from Theorem $8^{\prime}$ that either (i) $\operatorname{dim} N=2$ (and $H^{*}$ contains no particular vector), or (ii) $\operatorname{dim} N=4$ and $H^{*}=H^{(0)}$, or (iii) there is a direct sum decomposition $H^{*}=H_{1} \cup \cdots \cup H_{l}$, where each $H_{i}$ is of type (i) or (ii).

In case (i) all the vectors $v \in H \sim L$ lie in one two-dimensional plane $N^{\prime}$, and, consequently, $H \subset L \cup N^{\prime}$, that is, $H$ is decomposable, contradicting the condition of the theorem. In case (ii) the vector system $H \sim L$ lies in a four-dimensional subspace $N^{\prime}$ and is isomorphic to $H^{(0)}$. Consequently, $H \subset L \cup N^{\prime}$, i.e., $H$ is decomposable too, contradicting the condition of the theorem. The last case can be handled in the same way as cases (i) and (ii). This shows that in the case $\operatorname{dim} N \geq 2$ we have md $H^{*}=1$.

It follows from the previous proofs that (in the case $1 \leq \operatorname{dim} L<d$, that is, $\operatorname{dim}$ $N>0) H^{*}=\left\{e_{k+1}^{*}, \ldots, e_{d}^{*},-e_{k+1}^{*}, \ldots,-e_{d}^{*}\right\}$, where $e_{k+1}^{*}, \ldots, e_{d}^{*}$ is a basis in $N$. Let us denote by $G_{l}$ the subspace $\operatorname{lin}\left(L \cup \pi^{-1}\left(e_{l}^{*}\right)\right), l=k+1, \ldots, d$. Each subspace $G_{l}$ has the dimension $k+1$, and the inclusion $H \subset G_{k+1} \cup \cdots \cup G_{d}$ is true. Since $e_{i}$ is a particular vector, then there exists a hyperplane $Q_{i} \subset R^{d}$ through the origin, such that $H \sim\left\{e_{i}\right\} \subset P_{i}$ and $e_{i} \notin P_{i}$, where $P_{i}$ is a closed half-space with boundary hyperplane $Q_{i}(i=1, \ldots, k)$. By virtue of Lemma 9 , we have $e_{j} \in Q_{i}$ for $i \neq j$. The intersection $Q_{i} \cap G_{l}$ is a hyperplane in the space $G_{l}$, such that $e_{j} \in Q_{i} \cap G_{l}$ for $i \neq j$ and $e_{i} \notin Q_{i} \cap G_{l}$. Hence, the intersection $Q_{1} \cap \cdots \cap Q_{k} \cap G_{l}$ is a line which 
is not contained in $L$. We denote by $e_{l}$ a unit vector of this line. Then $e_{1}, \ldots, e_{k}$, $e_{l}$ is a basis of the subspace $G_{l}$, and, consequently, $e_{1}, \ldots, e_{k}, e_{k+1}, \ldots, e_{d}$ is a basis in $R^{d}$.

Since $H \sim\left\{e_{i}\right\} \subset P_{i}(i=1, \ldots, k)$, then each vector $v \in\left(H \cap G_{l}\right) \sim\left\{e_{i}\right\}$ has the form

$$
v=\alpha_{1} e_{1}+\cdots+\alpha_{k} e_{k}+\beta e_{l} \quad\left(\alpha_{i} \leq 0\right)
$$

Consequently, for each vector $v \in G_{l} \sim L$ we have

$$
-v=x_{1} e_{1}+\cdots+x_{k} e_{k}+x_{l} e_{l} \quad\left(x_{i} \geq 0 \text { for all } i=1, \ldots, k\right) \text {. }
$$

Now we denote by $S_{l}^{(+)}, S_{l}^{(-)}$the half-spaces of all vectors

$$
x_{1} e_{1}+\cdots+x_{k} e_{k}+x_{k+1} e_{k+1}+\cdots+x_{d} e_{d}
$$

for which $x_{l} \geq 0$ and $x_{l} \leq 0$, respectively, and by $Q_{l}$ their intersection, that is, $Q_{l}=S_{l}^{(+)} \cap S_{l}^{(-)}$is the $(d-1)$-dimensional subspace spanning all vectors $e_{1}, \ldots, e_{d}$ except $e_{l}(l$ is one of the indices $k+1, \ldots, d)$. Then $G_{l} \cap S_{l}^{(+)}$and $G_{l} \cap S_{l}^{(-)}$are two closed half-spaces in $G_{l}$ with the common boundary hyperplane $L \subset G_{l}$. It is easily shown that each of the sets $H_{l}^{(+)}=H \cap G_{l} \cap$ int $S_{l}^{(+)}=H \cap$ int $S_{l}^{(+)}, H_{l}^{(-)}=$ $H \cap G_{l} \cap$ int $S_{l}^{(-)}=H \cap \operatorname{int} S_{l}^{(-)}$contains, at least, two elements. Indeed, if $H \cap$ int $S_{l}^{+)}$contains no element of $H$, then the system $H$ is one-sided (contradicting the condition of the theorem), and if $H \cap$ int $S^{(+)}$contains only one element $e$ of $H$, then $e$ is a particular element of $H$ (contradicting the relation $e \notin L$ ). Hence, $H \cap$ int $S^{(+)}$contains, at least, two elements of $H$. Similarly, $H \cap$ int $S^{(-)}$contains, at least, two elements of $H$.

Let now $v \in H_{l}^{(+)}, v^{\prime} \in H_{l}^{(-)}$, that is, $-\alpha v=x_{1} e_{1}+\cdots+x_{k} e_{k}+e_{l},-\alpha^{\prime} v^{\prime}=$ $x_{1}^{\prime} e_{1}+\cdots+x_{k}^{\prime} e_{k}-e_{l}$, where $\alpha>0, \alpha^{\prime}>0$ and all the coefficients $x_{1}, \ldots, x_{k}$, $x_{1}^{\prime}, \ldots, x_{k}^{\prime}$ are nonnegative. Then

$$
\alpha v+\alpha^{\prime} v^{\prime}+\left(x_{1}+x_{1}^{\prime}\right) e_{1}+\cdots+\left(x_{k}+x_{k}^{\prime}\right) e_{k}=0
$$

and, consequently, at most, one of the sums $x_{1}+x_{1}^{\prime}, \ldots, x_{k}+x_{k}^{\prime}$ is positive (since $v, v^{\prime}, e_{1}, \ldots, e_{k} \in H$ and $\mathrm{md} H=2$ ). It follows from this that each vector $v \in H_{l}^{(+)}$ has the form $-\alpha v=x_{i} e_{i}+e_{l}$, where $i$ is one of the indices $1, \ldots, k$ and $\alpha>0$, $x_{i} \geq 0$. Moreover, there is at least one element $v \in H^{+)}$for which one of the coefficients $x_{1}, \ldots, x_{k}$ is positive, say, $-\alpha v=x_{i} e_{i}+e_{l}, x_{i}>0$ (since there are, at least, two elements of the set $H_{l}^{(+)}$, and if $x_{1}=\cdots=x_{k}=0$ for one of them, then we may take another element). Similarly, each vector $v^{\prime} \in H^{(-)}$has the form $-\alpha^{\prime} v^{\prime}=x_{j}^{\prime} e_{j}-e_{i} ; x_{j}^{\prime} \geq 0, \alpha^{\prime}>0$, and there is an element $v^{\prime} \in H_{l}^{(-)}$for which $x_{j}^{\prime}>0$. Finally, we conclude that $-\alpha v=x_{i} e_{i}+e_{l},-\alpha^{\prime} v^{\prime}=x_{j}^{\prime} e_{j}-e_{l}$, where the index $i=j$ is the same for all elements $v, v^{\prime}$. We denote this index by $\varphi(l)$. So we have $-\gamma w=x_{\varphi(l)} e_{\varphi(l)} \pm e_{l}$ for each $w \in\left(H \cap G_{l}\right) \sim L$, where $\varphi(l)$ is one of the numbers $1, \ldots, k$ and the coefficients $y>0, x_{\varphi(l)} \geq 0$ depend on $w$. This means that 
$H \sim L \subset$ norm $\left(\bigcup_{l>k} \sqcap_{l, \varphi(l)}\right)$ (see the statement of Theorem 9). More detailed,

$$
\begin{aligned}
& H \sim L \subset \text { norm } \bigcup_{l>1} \Pi_{i, 1} \quad \text { for } \quad k=1, \\
& H \sim L \subset \text { norm } \bigcup_{l>k} \Pi_{l, \varphi(l)} \quad \text { for } \quad 2 \leq k \leq d-1, \\
& H \sim L=\varnothing \quad \text { for } \quad k=d
\end{aligned}
$$

(see (1)-(4)).

It remains to investigate the part $H \cap L$ of the vector system $H$. We investigate this point in the general case, that is, for $\operatorname{dim} N=0,1, \ldots, d-1$ (or, what is the same, for $k=\operatorname{dim} L=1, \ldots, d)$. Since $H \sim\left\{e_{i}\right\} \subset P_{i}$ for $i=1, \ldots, k$, then

$$
\begin{aligned}
& (H \cap L) \sim\left\{e_{1}, \ldots, e_{k}\right\} \subset P_{1} \cap \cdots \cap P_{k} \cap L \\
& \quad=\left\{v:-v=x_{1} e_{1}+\cdots+x_{k} e_{k} ; x_{1} \geq 0, \ldots, x_{k} \geq 0\right\}
\end{aligned}
$$

This means that

$$
\begin{aligned}
& H \cap L \subset \operatorname{norm}\left\{e_{1},-e_{1}\right\} \quad \text { for } \quad k=1, \\
& H \cap L \subset \operatorname{norm}\left(\left\{e_{1}, \ldots, e_{k}\right\} \cup\left(\bigcup_{i<j \leq k} F_{i j}\right)\right) \quad \text { for } \quad 2 \leq k \leq d .
\end{aligned}
$$

Uniting the results obtained, we have, for the set $H=(H \cap L) \cup(H \sim L)$,

$$
\begin{aligned}
& H \subset \operatorname{norm}\left(\left\{e_{i}\right\} \cup\left(\bigcup_{l>1} \Pi_{l, 1}\right)\right) \quad \text { for } k=1, \\
& H \subset \operatorname{norm}\left(\left\{e_{1}, \ldots, e_{k}\right\} \cup\left(\bigcup_{i<j \leq k} F_{i j}\right) \cup\left(\bigcup_{l>k} \Pi_{l, \varphi(l)}\right)\right) \quad \text { for } 2 \leq k \leq d-1, \\
& H \subset \operatorname{norm}\left(\left\{e_{1}, \ldots, e_{d}\right\} \cup\left(\bigcup_{i<j \leq d} F_{i j}\right)\right) \quad \text { for } k=d .
\end{aligned}
$$

We notice, furthermore, that in the case $k=2$, if $\varphi$ is not onto, say, $\varphi(l)=1$ for all $l=3, \ldots, d$, then

$$
H \subset \operatorname{norm}\left(\left\{e_{1}, e_{2}\right\} \cup F_{12} \cup\left(\bigcup_{l>2} \Pi_{l, 1}\right)\right) \subset \operatorname{norm}\left(\left\{e_{1}\right\} \cup\left(\bigcup_{l>1} \Pi_{l, 1}\right)\right)
$$

(since $\left.\left\{e_{2}\right\} \cup F_{12} \subset \Pi_{2,1}\right)$. This means that for $k=2$ it is sufficient to consider the case when $\varphi$ is onto (see (3)).

It remains to prove that (i) md $H^{\prime}=2$ for every vector system $H^{\prime}$ indicated on the right-hand side of relations (1)-(4); (ii) all the 2 -systems indicated on the 
right-hand sides of relations (1)-(4) are maximal; and (iii) all the 2-systems on the right-hand sides of relations (1)-(4) are indecomposable.

Let us prove assertion (i). Let $a_{0}, \ldots, a_{m} \in H^{\prime}$ be minimally dependent vectors. First we remark that for every $j=1, \ldots, d$ the intersection $H^{\prime} \cap Q_{j}$ is a vector system which is contained in a vector system of the same type as (1)-(4), but situated in $(d-1)$-dimensional space $Q_{j}$ (we write "is contained" instead of "coincides" because, for example, in the case $k=1$ the intersection $H^{\prime} \cap Q_{1}$ has the form $\left\{e_{2}, \ldots, e_{d},-e_{2}, \ldots,-e_{d}\right\}$, that is, $\left.\operatorname{md}\left(H^{\prime} \cap Q_{1}\right)<2\right)$. Thus, by an obvious induction, we conclude that if $\left\{a_{0}, \ldots, a_{m}\right\} \subset Q_{j}$, then $\operatorname{md}\left\{a_{0}, \ldots, a_{m}\right\} \leq 2$ (that is, $m \leq 2$ ).

Furthermore, in the case $k<d$ we may suppose that each intersection $\left\{a_{0}, \ldots, a_{m}\right\} \cap$ int $S_{d}^{(+)},\left\{a_{0}, \ldots, a_{m}\right\} \cap$ int $S_{d}^{(-)}$is nonempty (otherwise we have $\left\{a_{0}, \ldots, a_{m}\right\} \subset Q_{d}$ and, consequently, $m \leq 2$ ). Without loss of generality we may suppose that $a_{0} \in$ int $S_{d}^{(+)}, a_{1} \in$ int $S_{d}^{(-)}$(and hence $a_{0}, a_{1} \in \prod_{d, \varphi(d)}$ ). Moreover, $e_{\varphi(\mathrm{d})} \in\left\{a_{2}, \ldots, a_{m}\right\}$ (otherwise $\left\{a_{0}, \ldots, a_{m}\right\} \subset P_{\varphi(d)}$ and, consequently, $\left\{a_{0}, \ldots, a_{m}\right\}$ $\subset Q_{\varphi(d)}$, that is, $m \leq 2$ ). However, in this case the vectors $a_{0}, a_{1}, e_{\varphi(d)}$ are linearly dependent, and, consequently, $m \leq 2$ too.

Finally, in the case $k=d$ there exist indices $i, j$, such that $\left\{a_{0}, \ldots, a_{m}\right\} \cap F_{i j} \neq \varnothing$ (otherwise $\left\{a_{0}, \ldots, a_{m}\right\} \subset\left\{e_{1}, \ldots, e_{d}\right\}$, contradicting the minimal dependence of the vectors $\left.a_{0}, \ldots, a_{m}\right)$. Without loss of generality we may suppose that $\left\{a_{0}, \ldots\right.$, $\left.a_{m}\right\} \cap F_{12} \neq \varnothing$ and $a_{0} \in F_{12}$. Then $e_{1} \in\left\{a_{0}, \ldots, a_{m}\right\}$ (otherwise $\left\{a_{0}, \ldots, a_{m}\right\} \subset Q_{1}$ and $m \leq 2$ ); similarly, $e_{2} \in\left\{a_{0}, \ldots, a_{m}\right\}$. However, in this case the vectors $a_{0}, e_{1}$, $e_{2}$ are linearly dependent and, consequently, $m \leq 2$.

So, $m \leq 2$ in any case, that is, md $H^{\prime} \leq 2$ (the inequality $m d H^{\prime} \geq 2$ is obvious).

Now we prove that all the 2-systems $H^{\prime}$ indicated on the right-hand sides of relations (1) (4) are maximal. Thus we must prove that if $w \notin H^{\prime}$, where $w \in R^{d}$ is a unit vector, then $\operatorname{md}\left(H^{\prime} \cup\{w\}\right)>2$. We have $-w=\alpha_{1} e_{1}+\cdots+\alpha_{d} e_{d}$ and, consequently, $w+\left|\alpha_{1}\right| f_{1}+\cdots+\left|\alpha_{d}\right| f_{d}=0$, where $f_{i}= \pm e_{i}$. Hence, no more than two of the coefficients $\alpha_{1}, \ldots, \alpha_{d}$ are nonzero (because $f_{i} \in H^{\prime}$ for all $i=1, \ldots, d$ ). So, $w=\alpha_{i} e_{i}+\alpha_{j} e_{j}$, where $i<j$ and $\alpha_{i} \neq 0, \alpha_{j} \neq 0$. We consider three cases:

(i) $i<j \leq k$. In this case at least one of the coefficients $\alpha_{i}, \alpha_{j}$ (say, $\alpha_{i}$ ) is positive (since $F_{i j} \subset H^{\prime}$ ), that is, $w=\alpha_{i} e_{i}+\left|\alpha_{j}\right| f_{j}$ where $\alpha_{i}>0$ and $f_{j}= \pm e_{j}$. If now $k>2$ and $\mu \leq k$ is an index distinct from $i$ and $j$, then the vectors $w,-f_{j}$, $e_{\mu},-e_{i}-e_{\mu}$ are minimally dependent. Furthermore, if $k=2$, then there exists an index $l=k+1, \ldots, d$, such that $\varphi(l)=i$ (see (3)). Then the vectors $w,-f_{j}, e_{l},-e_{i}-e_{l}$ are minimally dependent.

(ii) $i \leq k<j$. If $\varphi(j) \neq i$, then $w=\left|\alpha_{i}\right| f_{i}+\left|\alpha_{j}\right| f_{j}$ where $f_{i}= \pm e_{i}$ and $f_{j}= \pm e_{j}$. Consequently, the vectors $w,-f_{j}, e_{\varphi(j)},-f_{i}-e_{\varphi(j)}$ are minimally dependent. Furthermore, if $\varphi(j)=i$ and $k \geq 2$, then there is an index $\mu \leq k$, such that $\mu \neq i$. In this case $w=\alpha_{i} e_{i}+\left|\alpha_{j}\right| f_{j}$, where $\alpha_{i}>0$ and $f_{j}= \pm e_{j}$. Consequently, the vectors $w,-f_{j}, e_{\mu},-e_{i}-e_{\mu}$ are minimally dependent. Finally, if $\varphi(j)=i$ and $k=1$, then $d-k \geq 2$ (we recall that $d \geq 3$ ), and, consequently, there exists an index $v>k$, such that $v \neq j$ and $\varphi(v)=$ $\varphi(j)=i=1$. In this case $w=\alpha_{i} e_{i}+\left|\alpha_{j}\right| f_{j}$, where $\alpha_{i}>0$ and $f_{j}= \pm e_{j}$. Hence, the vectors $w,-f_{j},-e_{v}, e_{v}-e_{\varphi(v)}$ are minimally dependent. 
(iii) $k<i<j$. In this case $w=\left|\alpha_{i}\right| f_{i}+\left|\alpha_{j}\right| f_{j}$, where $f_{i}= \pm e_{i}$ and $f_{j}= \pm e_{j}$. If $\varphi(i) \neq \varphi(j)$, then the vectors $w,-f_{i}-e_{\varphi(i)},-f_{j}-e_{\varphi(j)}, e_{\varphi(i)}, e_{\varphi(j)}$ are minimally dependent. If $\varphi(i)=\varphi(j)$, then the vectors $w,-f_{i}-e_{\varphi(i)},-f_{j}-e_{\varphi(j)}$, $e_{\varphi(i)}$ are minimally dependent.

Thus in any case we have $\operatorname{md}\left(H^{\prime} \cup\{w\}\right)>2$, contradicting the assumption.

Finally, every two elements of these systems are contained in a minimal dependent system. This proves that all the four right-hand sides are really indecomposable. This completes the proof.

\section{Proof of Theorem 10}

The theorem is deduced from the following lemmas. One of them (Lemma 15) is evident, and the others are known in the theory of convex sets. A similar construction is contained in Kincses' paper [9].

Lemma 12. Let $M \subset R^{d}$ be a compact convex body and let $H(M)$ be the set of the outward normals at all its regular boundary points. For every $u \in H(M)$ we denote by $P(u)$ the closed half-space with outward normal $u$, such that $P(u)$ contains $M$ and bd $P(u)$ is a support hyperplane of $M$. Then the intersection $\bigcap_{u \in H(M)} P(u)$ coincides with $M$.

Lemma 13. Let $M \subset R^{d}$ be a compact convex body and let $H(M)=H_{1} \cup \cdots \cup H_{k}$. We denote by $N_{i}$ the intersection $\bigcap_{u \in H_{i}} P(u)$. Then all the bodies $N_{i}$ are closed (maybe noncompact) and $M=N_{1} \cap \cdots \cap N_{k}$.

Lemma 14. If a compact convex body $M \subset R^{d}$ is represented as the intersection $N_{1} \cap \cdots \cap N_{k}$, where the convex bodies $N_{1}, \ldots, N_{k}$ are closed and $N_{1}^{*}, \ldots, N_{k}^{*}$ are compact, then $M^{*}=\operatorname{conv}\left(N_{1}^{*} \cup \cdots \cup N_{k}^{*}\right)$.

Lemma 15. If $H=H(M)$ satisfies conditions (1), (2), (3), or (4), then the body $M$ satisfies conditions $(\mathrm{A}),(\mathrm{B}),(\mathrm{C} ; k=2)$, and $(\mathrm{C} ; k>2)$ of Theorem 10 , respectively.

Now the "only in" part of Theorem 10 follows immediately from Lemmas 12-15. Indeed, if $H(M)$ contains at least one particular vector, then

$$
H(M)=\bigcup_{i<j \leq k}\left(H(M) \cap\left(\left\{e_{i}, e_{j}\right\} \cup F_{i j}\right)\right) \cup \bigcup_{l>k}\left(H(M) \cap\left(\left\{e_{l}, e_{\varphi(l)}\right\} \cup \Pi_{l, \varphi(l)}\right)\right) .
$$

The "in" part of Theorem 10 follows from the next evident lemma and Theorem 6.

Lemma 16. Let $N_{1}, \ldots, N_{k}$ be compact convex sets in $R^{d}$. Then

$$
\operatorname{ext} \operatorname{conv}\left(N_{1} \cup \cdots \cup N_{k}\right) \subset\left(\operatorname{ext} N_{1}\right) \cup \cdots \cup\left(\operatorname{ext} N_{k}\right)
$$




\section{Acknowledgments}

The author thanks Professors Victor Klee and Branko Grünbaum and also Mary Sheetz for kind terms and friendly help. The author thanks Professor János Kincses as well, whose kind, attentive, and careful reading of the text and whose essential comments improved the paper substantially.

\section{References}

1. B. Szökefalvi-Nagy, Ein Satz über Parallelverschiebungen konvexer Körper, Acta Sci. Math. 15 (1954), no. 3-4, 169-177.

2. V. G. Boltyanski, Generalization of a certain theorem of Szökefalvi-Nagy, Dokl. Akad. Nauk SSSR 228 (1976), no. 2, 265-268 (in Russian).

3. E. D. Baladze, A complete solution of the Szökefalvi-Nagy problem for zonohedra, Soviet Math. Dokl. 34 (1987), no. 3, 458 461.

4. E. D. Baladze, A solution of the Szökefalvi-Nagy problem for zonoids, Dokl. Akad. Nauk SSSR 310 (1990), 11-14 (English translation in Soviet Math. Dokl. 41 (1990)).

5. A. D. Alexandrov, A theorem on convex polyhedra, Trudy Mat. Inst. Steklov Sect. Math 4 (1933), 87 (in Russian).

6. V. A. Zalgaller, and $\mathrm{Yu}$. G. Reshetnyak, On rectifiable curves, additive vector functions and displacement of segments, Vestnik Leningrad Univ. Fiz. Kim. 9 (1954), no. 2, 45-67 (in Russian).

7. E. D. Bolker, A class of convex bodies, Trans. Amer. Math. Soc. 145 (1969), 323-345.

8. V. G. Boltyanski and T. A. Chabukiani, Solution of the Szökefalvi-Nagy problem for threedimensional convex bodies, Dokl. Adad. Nauk SSSR 279 (1984), no. 5, 1033-1035 (in Russian).

9. J. Kincses, The classification of 3- and 4-Helly dimensional convex bodies, Geom. Dedicata 22 (1987), 283-301.

10. V. G. Boltyanski, Helly's theorem for H-convex sets, Dokl. Akad. Nauk SSSR 226 (1976), no. 2, 249-252 (in Russian).

11. R. Givalevich, Publ. Inst. Math. 26 (1979), 307-311.

Received August 20, 1990, and in revised form December 13, 1991. 